\title{
Lignin in the humic acids of Chernozems of Transbaikalia
}

Chimitdorzhieva E.O., Chimitdorzhieva G.D.

Institute of General and Experimental Biology SB RAS, 6 Sakhyanovoi st., Ulan-Ude, 670047, Russia, erzhena_ch@mail.ru, galdorj@gmail.com

Keywords: lignin, humic acids, lignin phenols, dispersed carbonate chernozem, quasigley chernozem doi: 10.36291/HIT.2019.chimitdorzhieva.024

Lignin structures are known to be included to the aromatic core of humic acids (HA), which form the organic part of soils (Fleig, 1971; Manskaya, Kodina, 1975; Tuev, 1989; Orlov, 1990; Chukov, 2001; Zavarzin, 2004). Lignin of different plant species has a varied chemical structure and molecular weight (Fang et al., 2017). Features of the climate of Transbaikalia: negative mean annual temperature $\left(-4^{0} \mathrm{C}\right)$, soils for a long time (5-7 months) and deep (up to 3-4m) frozen, short vegetation period, summer is short but hot, high solar radiation level (up to 3000 hours / year ), low precipitation (200-300 mm / year), large fluctuations in the mean daily and monthly air temperatures. Such extreme conditions promote a high accumulation of stable carbon compounds in the form of lignin (up to 35\%) in herbaceous plants as adaptation reaction. Grasses of Transbaikalia are peculiar, due to the imposition of latitudinal and altitudinal zonality. Hence, the aim of the study was to identify the quantity and quality of lignin phenols in herbs and their transformation into humic acids. The objects of the study are grassy vegetation and humic acids (HA) from the disperse-carbonate Chernozems of the Tugnuy Hollow of the Selenginsky Middle Mountain and quasigley Chernozems of the South part of the Vitim Plateau. Lignin was determined by mild alkaline oxidation of copper oxide in a nitrogen medium (Ertel, Hedges, 1984) at the Hanover University, Germany, with the advice of professors Gugenberger, and W. Zech from Bayreuth University. While determining lignin parameters in grasses from two subtypes of Chernozems, we obtained: 1) vanillin phenols are accumulated more intensive in herbs on permafrost Chernozems compared to steppe ones; 2) lilac phenols content is higher in vegetation from permafrost Chernozems too; 3 ) compositional ratio $\mathrm{V}$ : $\mathrm{S}$ : $\mathrm{C}$ of phenols in plants on permafrost soils was following: for forbs 5: 5: 1; for legumes 5: 3: 1 ; for cereals 1: $1: 1$, and from steppe Chernozems, respectively: for forbs 2: 3: 1; for legumes 3: 2: 1; for cereals 2: 1: 1 . It would seem that soil hydromorphism is more conducive to the accumulation of natural phenols in vegetation, but this is not noticeable in a $0-10 \mathrm{~cm}$ layer of quasigley Chernozems, where $\mathrm{V}: \mathrm{S}: \mathrm{C}=2$ : 1: 1 versus from steppe one: 3: 3: 1. HA from permafrost soils contain significant amount of both vanillin and lilac structures, where $\mathrm{V}: \mathrm{S}$ : $\mathrm{C}=5: 4: 1$, while in HA from steppe soils this ratio is $3: 4: 1$. HA from both soils contain the same number of lilac structures that requires additional research. It should be assumed that initially high lignin content in vegetation should have contributed to a high accumulation of lignin in soil and, accordingly, in HA from permafrost soils. But the results show that there are more lignin phenols in HA than in soil. It should be assumed: 1) part of the aromatic fragments of lignin is included in the structure of the HA molecules; 2) most part of lignin transfer to humine, which reaches up to $71 \%$ of the total carbon in quasigley Chernozems; 3 ) other part remains in soil detritus (Chimitdorzhieva, 2016).

Preliminary data do not allow to explain the results because of a large complex of factors (climatic conditions, physical and chemical properties of soils, unstable redox potential, cryoarid microflora, grass species diversity, high solar radiation, and many others).

This work was carried out under the theme of the state assignment No. AAAA-A 17117011810038-7. 